\title{
MET Exon 14 Mutations in Non-Small-Cell Lung Cancer Are Associated With Advanced Age and Stage-Dependent MET Genomic Amplification and c-Met Overexpression
}

\section{Citation}

Awad, Mark M., Geoffrey R. Oxnard, David M. Jackman, Daniel O. Savukoski, Dimity Hall, Priyanka Shivdasani, Jennifer C. Heng, et al. 2016. "MET Exon 14 Mutations in Non-SmallCell Lung Cancer Are Associated With Advanced Age and Stage-Dependent MET Genomic Amplification and c-Met Overexpression." Journal of Clinical Oncology 34 (7) (March): 721-730. doi:10.1200/jco.2015.63.4600.

\section{Published Version}

doi:10.1200/JC0.2015.63.4600

\section{Permanent link}

http://nrs.harvard.edu/urn-3:HUL.InstRepos:32705575

\section{Terms of Use}

This article was downloaded from Harvard University's DASH repository, and is made available under the terms and conditions applicable to Other Posted Material, as set forth at http:// nrs.harvard.edu/urn-3:HUL.InstRepos:dash.current.terms-of-use\#LAA

\section{Share Your Story}

The Harvard community has made this article openly available.

Please share how this access benefits you. Submit a story.

\section{Accessibility}




\section{MET Exon 14 Mutations in Non-Small-Cell Lung Cancer Are Associated With Advanced Age and Stage-Dependent MET Genomic Amplification and c-Met Overexpression}

Mark M. Awad, Geoffrey R. Oxnard, David M. Jackman, Daniel O. Savukoski, Dimity Hall, Priyanka Shivdasani, Jennifer C. Heng, Suzanne E. Dahlberg, Pasi A. Jänne, Suman Verma, James Christensen, Peter S. Hammerman, and Lynette M. Sholl

Listen to the podcast by Dr Doebele at www.jco.org/podcasts

Mark M. Awad, Geoffrey R. Oxnard, David M. Jackman, Jennifer C. Heng, Suzanne E. Dahlberg, Pasi A. Jänne, and Peter S. Hammerman, Dana-Farber Cancer Institute; Mark M. Awad, Geoffrey R. Oxnard, David M. Jackman, Daniel O. Savukoski, Dimity Hall, Priyanka Shivdasani, Pasi A. Jänne, Peter S. Hammerman, and Lynette M. Sholl, Brigham and Women's Hospital and Harvard Medical School, Boston, MA; Suman Verma, ResearchDX, Irvine; and James Christensen, Mirati Therapeutics, San Diego, CA.

Published online ahead of print at www.jco.org on January 4, 2016

Authors' disclosures of potential conflicts of interest are found in the article online at www.jco.org. Author contributions are found at the end of this article.

Corresponding author: Mark M. Awad, $\mathrm{MD}, \mathrm{PhD}$, Dana-Farber Cancer Institute, 450 Brookline Ave, D1240G, Boston, MA 02215; e-mail: mark_awad@dfci.harvard. edu.

(c) 2016 by American Society of Clinical Oncology

0732-183X/16/3407w-721w/\$20.00 DOI: $10.1200 / J C 0.2015 .63 .4600$

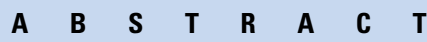

\section{Purpose}

Non-small-cell lung cancers (NSCLCs) harboring mutations in MET exon 14 and its flanking introns may respond to c-Met inhibitors. We sought to describe the clinical, pathologic, and genomic characteristics of patients with cancer with MET exon 14 mutations.

\section{Patients and Methods}

We interrogated next-generation sequencing results from 6,376 cancers to identify those harboring MET exon 14 mutations. Clinical characteristics of MET exon 14 mutated NSCLCs were compared with those of NSCLCs with activating mutations in KRAS and EGFR. Co-occurring genomic mutations and copy number alterations were identified. c-Met immunohistochemistry and real-time polymerase chain reaction to detect exon 14 skipping were performed where sufficient tissue was available.

\section{Results}

MET exon 14 mutations were identified in 28 of 933 nonsquamous NSCLCs (3.0\%) and were not seen in other cancer types in this study. Patients with MET exon 14-mutated NSCLC were significantly older (median age, 72.5 years) than patients with EGFR-mutant (median age, 61 years; $P<.001$ ) or KRAS-mutant NSCLC (median age, 65 years; $P<.001$ ). Among patients with METexon 14 mutations, 68\% were women, and 36\% were never-smokers. Stage IV MET exon 14-mutated NSCLCs were significantly more likely to have concurrent METgenomic amplification (mean ratio of MET to chromosome 7,4.3) and strong c-Met immunohistochemical expression (mean H score, 253) than stage IA to IIIB MET exon 14-mutated NSCLCs (mean ratio of MET to chromosome 7, 1.4; $P=.007$; mean H score, 155; $P=.002$ ) and stage IV MET exon 14-wild-type NSCLCs (mean ratio of MET to chromosome 7, 1.2; $P<.001$; mean $\mathrm{H}$ score, $142 ; P<.001$ ). A patient whose lung cancer harbored a MET exon 14 mutation with concurrent genomic amplification of the mutated MET allele experienced a major partial response to the c-Met inhibitor crizotinib.

\section{Conclusion}

MET exon 14 mutations represent a clinically unique molecular subtype of NSCLC. Prospective clinical trials with c-Met inhibitors will be necessary to validate MET exon 14 mutations as an important therapeutic target in NSCLC.

\section{J Clin Oncol 34:721-730. (C) 2016 by American Society of Clinical Oncology}

\section{INTRODUCTION}

In the past decade, the discovery of targetable genomic alterations in non-small cell-lung cancer (NSCLC) has revolutionized treatment of patients whose tumors harbor mutations in genes such as EGFR, ${ }^{1-3} A L K,{ }^{4-6}$ and ROS1. ${ }^{7,8}$ More recently, activating mutations and genomic amplification in the mesenchymal-to-epithelial transition
(MET) gene have been recognized as a potentially important therapeutic target in NSCLC. ${ }^{9-14}$ With a number of c-Met inhibitors already in clinical use, ${ }^{15}$ prospective identification of MET genomic alterations may help guide treatment of a subset of patients with lung cancer toward more effective genotype-directed therapies.

c-Met is the tyrosine kinase receptor for hepatocyte growth factor. The intracellular c-Met juxtamembrane domain is encoded in part by 
MET exon 14 and contains critical regulatory elements, including tyrosine 1003, the direct binding site for Cbl, an E3 ubiquitin ligase that promotes c-Met protein degradation. ${ }^{16}$ First recognized in NSCLC more than 10 years ago, somatic mutations in the MET gene can cause exon 14 skipping, and the resulting mutant receptor demonstrates increased c-Met signaling and oncogenic potential. ${ }^{17,18}$

With the advent of improved sequencing technologies, routine detection of MET exon 14 mutations has become more feasible. Several recent reports have shown that patients with MET exon 14-mutant NSCLC may respond to treatment with c-Met inhibitors such as crizotinib and cabozantinib. ${ }^{9-13}$ In our study, we screened a large cohort of diverse tumor types at our institution and found MET exon 14 mutations in 28 patients with NSCLC. Here, we describe the unique clinical, molecular, and pathologic characteristics of this cohort and describe the case of a patient who achieved a major partial response to crizotinib.

\section{PATIENTS AND METHODS}

\section{Study Population}

The study population was composed of patients at the Dana-Farber Cancer Institute who consented to an institutional review board-approved prospective cohort study for cancer sequencing between August 1, 2013, and May 1, 2015. ${ }^{19,20}$ Demographic and clinical characteristics were collected from participants who had provided written informed consent for a separate institutional review board-approved clinical research protocol.

\section{Next-Generation Sequencing}

Next-generation sequencing (NGS) performed on 282 cancer-related genes (Appendix Table A1, online only) is described in the supplemental methods section. Because criteria for copy-number cutoffs by NGS have not been well established, a modified approach to the clinically validated fluorescent in situ hybridization-based criteria for amplification used in our laboratory ( $\geq 3: 1$ ratio of MET to CEP7) was applied. High-level gene copy gain (amplification) required evidence for focal $M E T$ gain at a ratio of 3:1 or greater of $M E T$ to chromosome 7, defined as the mean copy number of the $M E T$ gene (averaged across all the sequenced exons and targeted introns of the MET gene) normalized against the mean copy number of chromosome 7 (excluding regions of focal high amplification or deep deletions affecting other genes). Low copy gain was defined as a MET to chromosome 7 ratio of greater than 1 and less than 3 . These criteria were similarly applied to other analyzed genes in this study.

\section{Immunohistochemistry}

A board-certified pathologist with expertise in thoracic malignancies (L.M.S.) classified each tumor histology according to WHO and International Association for the Study of Lung Cancer guidelines. ${ }^{21,22}$ Immunohistochemical (IHC) staining for c-Met, ALK, and ROS1 was performed on formalin-fixed paraffin-embedded tissue sections $4 \mu \mathrm{m}$ in thickness and scored using published criteria. ${ }^{23-26}$ Clone names and staining conditions are listed in Appendix Table A2 (online only). Staining for c-Met was scored semiquantitatively on a four-tier scale from 0 (absent) to 3 (strong membranous and cytoplasmic staining). Intensity scores were multiplied by percentage of tumor cells staining to generate an $\mathrm{H}$ score (maximum score, 300).

\section{Qualitative Real-Time Polymerase Chain Reaction for Detection of MET Exon 14 Skipping}

RNA was extracted from formalin-fixed paraffin-embedded samples per protocol (AllPrep DNA/RNA mini kit; Qiagen, Hilden, Germany) and quantitated using Molecular Probes Quant-iT RiboGreen RNA assay kit
(Life Technologies, Carlsbad, CA). FAM-labeled primer-probe sets specific for MET exon 14 deletion products and MET-wild-type products were combined with $10 \mathrm{ng}$ of RNA in individual reactions. Qualitative real-time polymerase chain reaction (qRT-PCR) of test samples and positive and negative controls with 40 amplification cycles was performed in duplicate on an ABI 7900 RT-PCR system (Life Technologies) according to manufacturer protocol (ResearchDX, Irvine, CA).

\section{Statistical Analysis}

Fisher's exact and Wilcoxon rank-sum tests were used to compare categorical and continuous variables, respectively. All reported $P$ values are two-sided hypothesis tests conducted at the .05 level, and no adjustments were made for multiple comparisons.

\section{RESULTS}

\section{Patient Characteristics}

To determine the frequency of MET exon 14 mutations in various cancer types, 6,376 solid and hematologic malignancies were analyzed using NGS between August 1, 2013, and May 1, 2015. Of the 1,141 lung cancers in this cohort, MET exon 14 mutations were identified in $28(3.0 \%)$ of 933 nonsquamous NSCLCs (Fig 1), comprising 873 adenocarcinomas, 15 pleomorphic carcinomas, and 45 NSCLCs, including poorly differentiated carcinomas, large-cell carcinomas, and adenosquamous carcinomas. MET exon 14 mutations were not detected in other tumor types or other lung cancer histologic subtypes, including 132

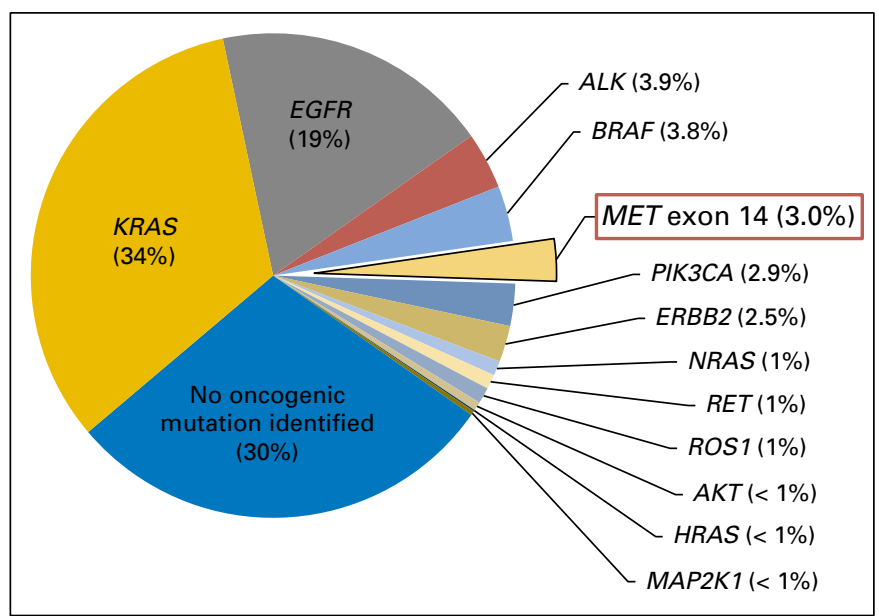

Fig 1. Distribution of genotypes among 933 patients with nonsquamous non-small-cell lung cancer (NSCLC). Results from next-generation sequencing of these 933 patient cases are shown. In this prevalence cohort, MET exon 14 mutations were detected in 28 patients with nonsquamous NSCLC (3.0\%). None of these 28 patients also had an activating mutation in KRAS, EGFR, or ERBB2 or a chromosomal rearrangement in $A L K, R O S 1$, or RET. Of the $35 B R A F$ mutations detected in the cohort, 16 involved codon V600. Seven BRAF mutations cooccurred with other oncogenic mutations, most often as a minor subclone. Of the 27 PIK3CA mutations detected in the cohort, 14 co-occurred with another oncogenic mutation, including KRAS, EGFR, and MET exon 14. NRAS Q61L mutations co-occurred with KRAS mutations in two patients, and a BRAFexon 11 mutation occurred in one patient. A MAP2K1 D67N variant co-occurred with a $K R A S$ and $A K T 1$ mutation in one patient each. A single patient had both EGFR L858R and ERBB2 extracellular domain (S310F) mutations. The figure represents the percentage of patients in the overall cohort with a functional variant in each gene; because of co-occurring mutations, the percentages total more than $100 \%$. 
squamous cell carcinomas, 41 SCLCs, 20 typical or atypical carcinoid tumors, and 15 neuroendocrine carcinomas, including large-cell neuroendocrine carcinomas and SCLC and large-cell neuroendocrine carcinoma combinations.

The clinical and pathologic characteristics of all 28 patients with MET exon 14-mutated NSCLC are listed in Table 1. Among these patients, the median age at disease onset was 72.5 years (range, 59 to 84 years), 19 (68\%) were women, and 10 (36\%) were never-smokers. At the time of their cancer diagnosis, 13 patients (46\%) with MET exon 14 mutations had stage I NSCLC, two (7\%) had stage II disease, four (14\%) had stage III disease, and nine (32\%) had stage IV disease. Histologic analysis showed that 18 patients $(64 \%)$ had adenocarcinoma, four (14\%) had pleomorphic (including sarcomatoid) carcinoma with an adenocarcinoma component, five (18\%) had poorly differentiated NSCLC not otherwise specified, and one (4\%) had adenosquamous histology. The four patients with pleomorphic or sarcomatoid histology and MET exon 14 mutations represented 26.7\% of 15 total patients with pulmonary sarcomatoid carcinoma sequenced in our cohort, consistent with a recent report that $M E T$ exon 14 mutations seem to be enriched in this histologic subtype of NSCLC. ${ }^{27}$

We compared demographic characteristics of the cohort of patients with $M E T$ exon 14 mutations with those of patients with NSCLCs harboring activating mutations in EGFR and KRAS identified during the same period of time who had also consented to our institutional clinical research protocol (Table 1). Patients with MET exon 14-mutant NSCLC were significantly older than patients with EGFR-mutant $(P<.001)$ and KRAS-mutant NSCLC $(P<.001)$. Patients with $M E T$ exon 14 mutations were significantly more likely than those with KRAS mutations to be never-smokers $(P<.001)$ and significantly more likely than those with EGFR mutations to have a history of tobacco use $(P=.03)$. Asian race was only enriched in the cohort of those with EGFR mutations $(P<$ .001 ), and all 28 patients in the MET exon 14 cohort were white, non-Hispanic. A significantly higher percentage of patients with $M E T$ exon 14 mutations had stage I disease compared with those with EGFR or KRAS mutations $(P<.001)$.

\section{Characterization of MET Exon 14 Mutations}

The positions of the MET mutations relative to MET exon 14 and its flanking introns are shown in Fig 2. Genomic deletions occurred in $17(61 \%)$ of the 28 patients with MET exon 14 mutations, ranging in size from a two-base pair deletion to a 193-base pair deletion, and point mutations occurred in 11 patients $(39 \%)$. Of the 17 deletions, four were entirely within intron 13 but did not disrupt the intron 13 splice acceptor site, six overlapped with the intron 13 splice acceptor site, two occurred entirely within exon 14 (one in frame, one out of frame), and five involved the splice donor site of intron 14. Of the 11 point mutations, one resulted in a Y1003C amino acid substitution at the $\mathrm{Cbl}$ binding site, seven disrupted the splice donor site of intron 14, and three occurred in the last nucleotide of exon 14 (c.3028G $>$ A); one of these latter three also had a splice acceptor mutation in intron 5 of unclear significance.

\begin{tabular}{|c|c|c|c|}
\hline \multirow[b]{2}{*}{ Characteristic } & \multicolumn{3}{|c|}{ No. $(\%)$} \\
\hline & $\begin{array}{l}\text { MET Exon } 14 \\
(\mathrm{n}=28)\end{array}$ & $\begin{array}{c}\text { EGFR } \\
(\mathrm{n}=99)\end{array}$ & $\begin{array}{c}\text { KRAS } \\
(n=169)\end{array}$ \\
\hline Median age (range), years & $72.5(59-84)$ & $61(30-93)$ & $65(42-93)$ \\
\hline \multicolumn{4}{|l|}{ Sex } \\
\hline Male & $9(32)$ & $30(30)$ & $62(37)$ \\
\hline Female & $19(68)$ & $69(70)$ & $107(63)$ \\
\hline \multicolumn{4}{|l|}{ Smoking history, pack-years* } \\
\hline Never-smoker & $10(36)$ & $57(58)$ & $6(4)$ \\
\hline$\leq 10$ & $3(11)$ & $10(10)$ & $11(7)$ \\
\hline$>10$ & $15(53)$ & $28(28)$ & $152(90)$ \\
\hline \multicolumn{4}{|l|}{ Race } \\
\hline White, non-Hispanic & $28(100)$ & $79(80)$ & $157(93)$ \\
\hline Asian & $0(0)$ & $15(15)$ & $0(0)$ \\
\hline Black & $0(0)$ & $1(1)$ & $5(3)$ \\
\hline White, Hispanic & $0(0)$ & $3(3)$ & $3(2)$ \\
\hline Unknown & $0(0)$ & $1(1)$ & $4(2)$ \\
\hline \multicolumn{4}{|l|}{ Histology } \\
\hline Adenocarcinoma & $18(64)$ & $92(93)$ & $150(89)$ \\
\hline Pleomorphic with adenocarcinoma component & $4(14)$ & $0(0)$ & $3(2)$ \\
\hline NSCLC, poorly differentiated & $5(18)$ & $4(4)$ & $10(6)$ \\
\hline Squamous & $0(0)$ & $2(2)$ & $5(3)$ \\
\hline Adenosquamous & 1 (4) & $1(1)$ & $1(1)$ \\
\hline \multicolumn{4}{|l|}{ Stage at diagnosis } \\
\hline I & $13(46)$ & $9(9)$ & $12(7)$ \\
\hline$\|$ & $2(7)$ & $3(3)$ & $12(7)$ \\
\hline III & $4(14)$ & $9(9)$ & $44(26)$ \\
\hline IV & $9(32)$ & 78 (79) & $101(60)$ \\
\hline
\end{tabular}




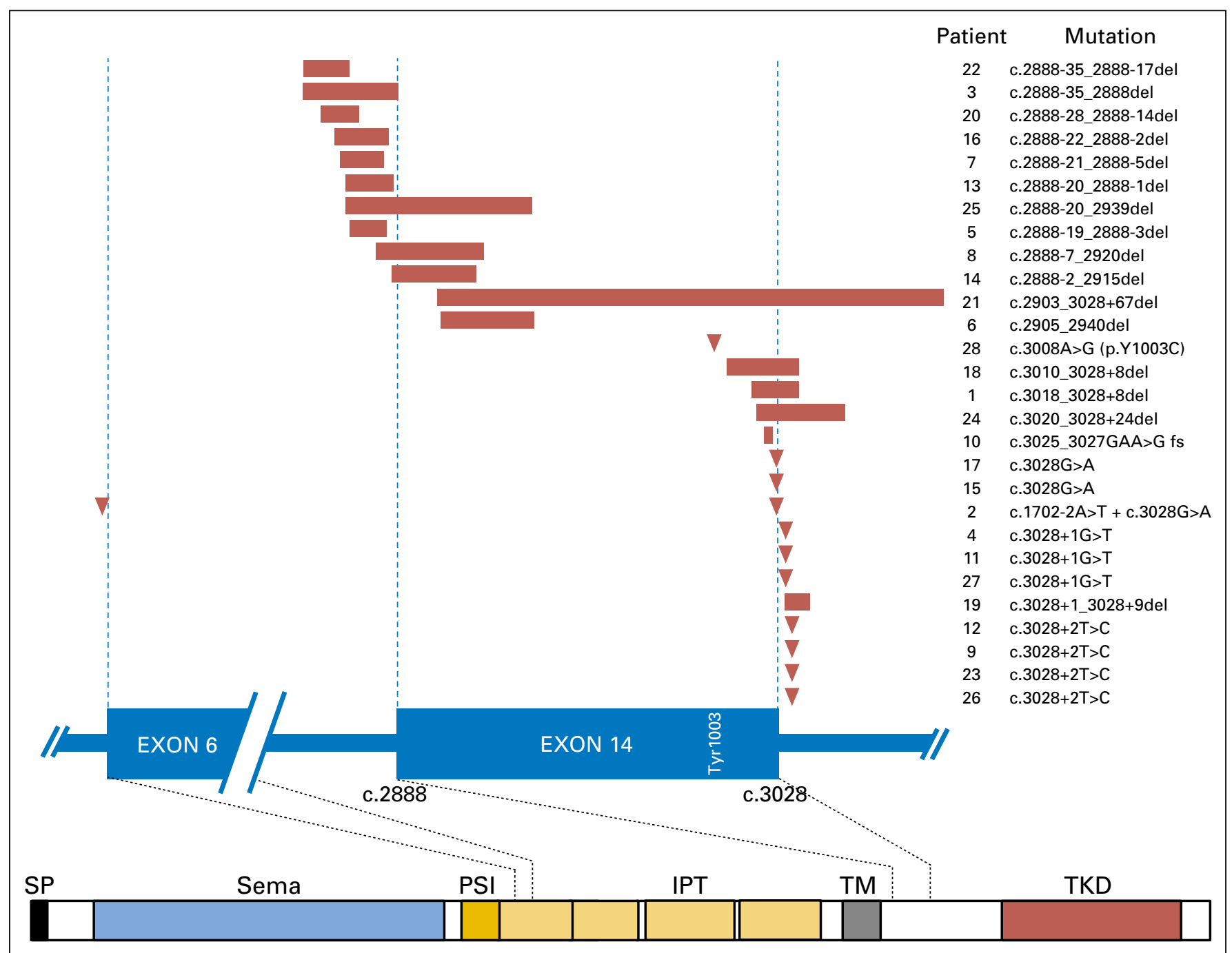

Fig 2. Locations of MET exon 14 genomic alterations found in 28 patients with non-small-cell lung cancer. The positions of each $M E T$ mutation are displayed in relation to

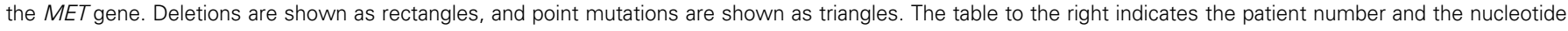

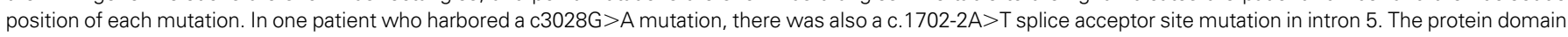

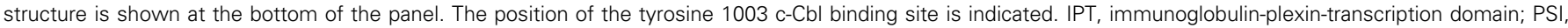

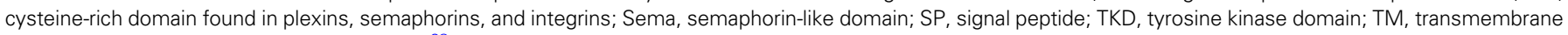
domain. c-Met protein diagram data adapted. ${ }^{28}$

\section{Concurrent Genomic Alterations}

None of the 28 patients with MET exon 14 mutations also had activating mutations in KRAS, EGFR, or ERBB2 or rearrangements in $A L K, R O S 1$, or RET (Figs 1 and 3). In one patient, a MET c3025_3027GAA $>$ G frame-shift mutation was detected in $76 \%$ of 453 sequencing reads, and there was also a concurrent $B R A F$ V600E mutation in $3 \%$ of 159 reads (Fig 3), suggesting that the $B R A F$ mutation occurred in a subclonal tumor population. EGFR copy gain was also observed in eight patients (29\%) with MET exon 14 mutations. Inactivating mutations in TP53 were observed in nine patients (32\%), and amplification of $M D M 2$, a negative regulator of $\mathrm{p} 53,{ }^{29}$ was observed in 13 patients (46\%). TP53 mutation and MDM2 amplification tended to occur in a nonoverlapping distribution, with a total of 20 patients $(71 \%)$ having alterations in at least one of these genes (Fig 3). Concurrent MDM2 amplification was significantly more common in $M E T$ exon 14-mutated lung cancers than in patients with KRAS (one of 315 [0.3\%]; $P<.001$ ) or EGFR mutations (six [3.4\%] of $178 ; P<.001$ ). Compared with patients with MET exon 14 mutations, concurrent TP53 mutations occurred at a similar frequency among those with KRAS mutations (113 of 315 [36\%]; $P=.84$ ) but were more common among those with EGFR mutations (112 [63\%] of 178; $P$ $=.003)$. Other selected co-occurring genomic mutations and copy number alterations are shown in Fig 3.

Genomic copy-number analysis demonstrated that among the 28 patients with $M E T$ exon 14 mutations, six (21\%) had concurrent high-level MET copy gain, and eight (29\%) showed low-level MET copy gain (Fig 3). In all 14 such patients, the MET exon 14-mutated allele seemed to be selectively amplified over the MET-wild-type allele on the basis of read count bias toward the mutant allele. Stage IV NSCLCs with MET exon 14 mutations had a significantly higher ratio of $M E T$ to chromosome 7 (mean ratio, 4.3) than stage I to III 


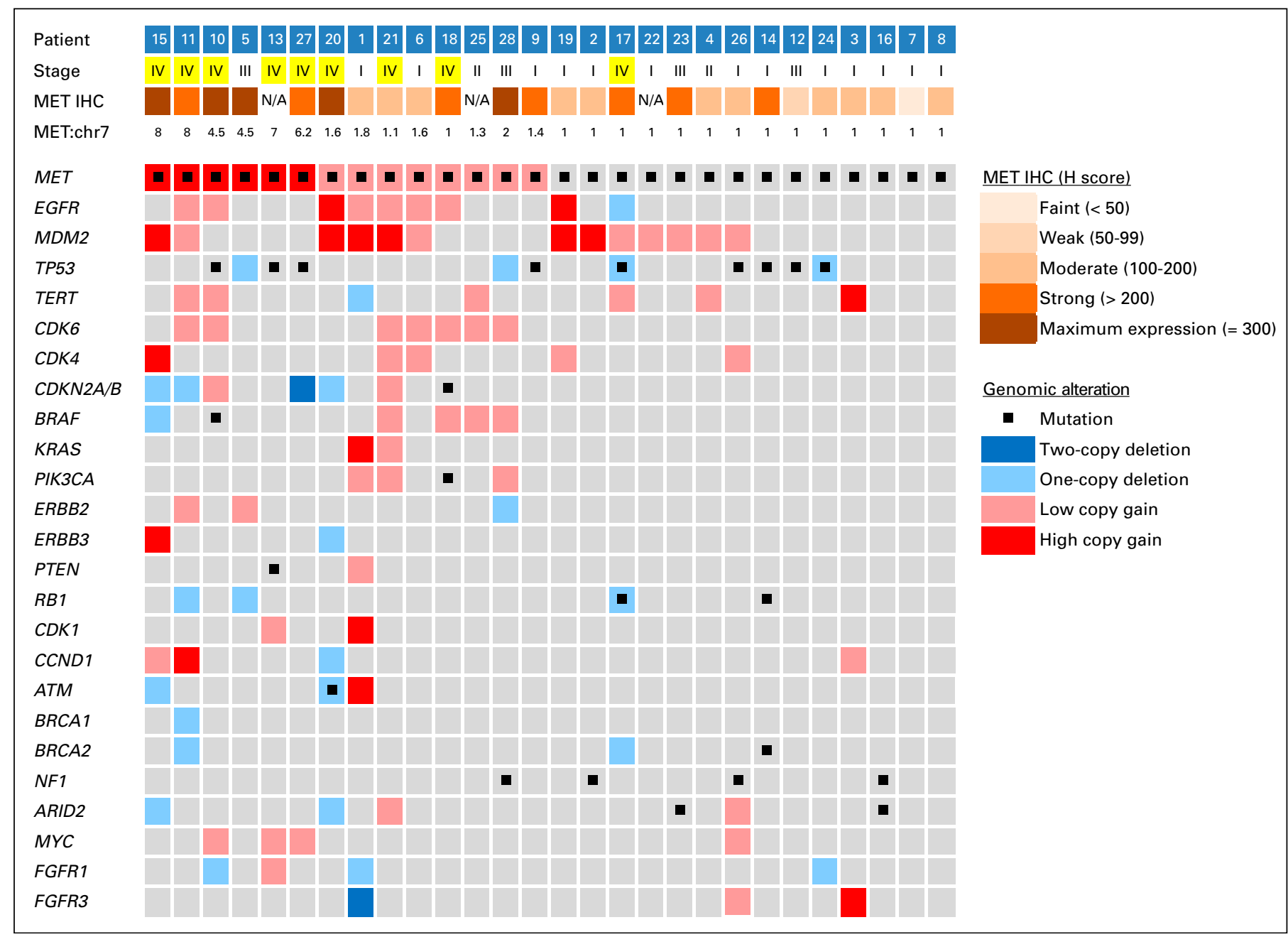

Fig 3. c-Met immunohistochemistry (IHC), co-occurring mutations, and copy-number alterations in tumors harboring MET exon 14 mutations. Patient numbers are shown in the top row. Stage at presentation is indicated, with patients with stage IV disease highlighted in yellow. c-Met IHC staining is shown in shades of brown reflecting the $\mathrm{H}$ score, which is the product of percent of positively staining cells (from 0 to 100 ) and the intensity of staining $(0,1+, 2+, 3+)$, with a maximum $\mathrm{H}$ score of 300. The copy-number ratios of MET to chromosome 7 (MET:chr7) are shown. Mutations are shown as black squares within each box. High-level amplification (MET:chr7 ratio $\geq 3$ ), red. Low-level amplification (MET:chr7 ratio $>1$ and $<3$ ), pink. One-copy deletion, light blue. Two-copy deletion, blue.

NSCLCs with MET exon 14 mutations (mean ratio of MET to chromosome $7,1.4 ; P=.007)$ and than 67 stage IV NSCLCs that lacked MET exon 14 mutations (mean ratio of MET to chromosome $7,1.2 ; P<.001$; Fig $4 \mathrm{~A}$ ).

\section{c-Met Expression by IHC}

Sufficient tissue to perform c-Met IHC was available for 25 of 28 patients (Fig 3). c-Met staining in the cohort of those with MET exon 14 mutations varied from weak expression $(\mathrm{H}$ score, $<50)$ to maximum expression (H score, 300). Stage IV NSCLCs with MET exon 14 mutations had a significantly higher $\mathrm{H}$ score (mean, 253) than stage I to III NSCLCs with MET exon 14 mutations (mean $\mathrm{H}$ score, $155 ; P=.002)$ and than 82 stage IV NSCLCs that lacked MET exon 14 mutations (mean $\mathrm{H}$ score, 142; $P<.001$; Fig 4B).

Representative histologic stains and genomic copy-number plots from a patient with stage I and a patient with stage IV NSCLC are shown in Figs 4C and 4D, respectively. Fig 4C shows patient 2, a never-smoker with stage I minimally invasive lung adenocarcinoma, lepidic predominant, with no MET genomic amplification (ratio of MET to chromosome 7, 1.0), moderate c-Met expression (H score, 120), focal low-level MDM2 amplification, and few other genomic alterations. In contrast, Fig 4D shows patient 20 , a patient with a 40 -pack-year smoking history with stage IV high-grade solid adenocarcinoma with high-level c-Met protein expression. This tumor showed marked genomic instability, with numerous copy-number alterations, including low-level amplification of EGFR and MET (ratio of MET to chromosome 7, 1.6), low-level gain of chromosome 8q, homozygous deletion of $C D K N 2 A / B$, and MDM2 amplification.

\section{MET Splicing Analysis}

We performed a qRT-PCR-based assay in patient samples where enough RNA was available for analysis. Exon 14 skipping was observed in 23 (96\%) of 24 samples (Appendix Fig A1A, online only), 


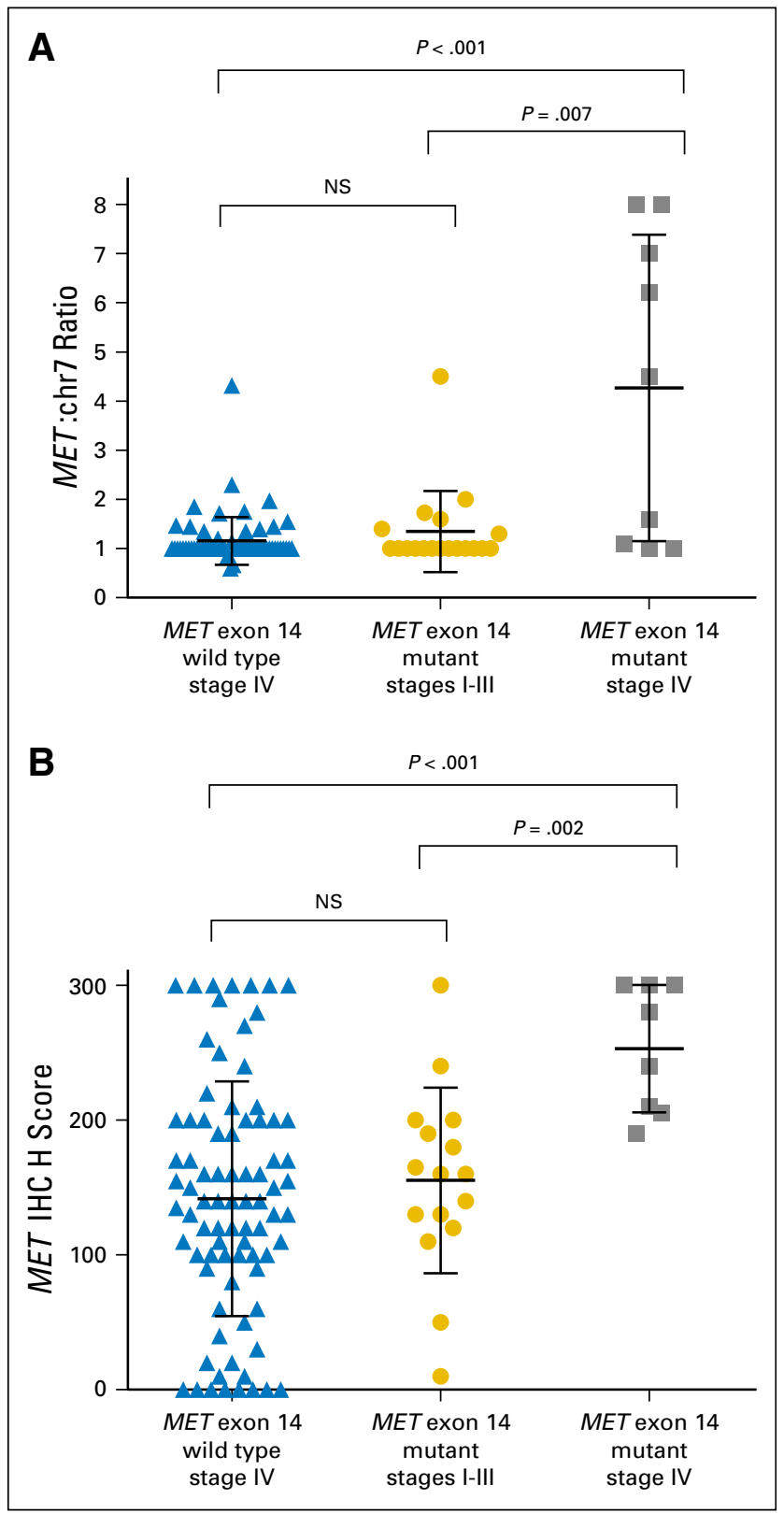

Fig 4. Histopathologic and genomic correlations with stage at presentation in patients with MET exon 14 mutations. Stage IV non-small-cell lung cancers (NSCLCs) with MET exon 14 mutations have significantly greater (A) MET amplification and (B) C-Met immunohistochemical (IHC) expression than stage IA to IIIB NSCLCs with MET exon 14 mutations and stage IV MET-wild-type NSCLCs. Error bars show mean values \pm one standard deviation. chr7, chromosome 7; NS, not significant.

including those that showed intronic deletions upstream of the intron 13 splice acceptor site, one with an in-frame deletion within exon 14, and those with a c.3028G $>$ A point mutation in the last nucleotide of exon 14. Exon skipping was not detected in any of the four control patients with lung cancer who lacked MET exon 14 mutations. In the one patient (patient 28) with a Y1003C substitution in the c-Cbl binding site, exon 14 skipping was not observed (Appendix Fig A1A); this patient's tumor displayed low-level MET genomic amplification (ratio of MET to chromosome 7,2) and maximal c-Met expression by IHC, with an $\mathrm{H}$ score of 300 (Fig 3; Appendix Fig A1B).

\section{Case Report: Response to Crizotinib}

A 64-year-old female never-smoker (patient 15) was diagnosed with stage IV NSCLC with poorly differentiated carcinoma histology, favoring adenocarcinoma. Her tumor underwent NGS at our institution, which showed no activating mutations in KRAS, EGFR, or $B R A F$ and no genomic rearrangements in ALK or ROS1. A c.3028G $>$ A mutation was identified in MET exon 14 in $94 \%$ of 867 reads (Fig 5A), in association with high-level MET amplification (Fig 5B). Exon 14 skipping was detected using qRT-PCR (Fig 5C). After disease progression during first-line chemotherapy, the patient started crizotinib $250 \mathrm{mg}$ orally twice per day, and repeat imaging 8 weeks later showed dramatic improvement in multiple lesions throughout her body (Figs 5D and 5E), with an ongoing response at 8 months.

\section{DISCUSSION}

Lung cancer remains the leading cause of cancer-related death worldwide, and detection of targetable genomic mutations within tumors will continue to improve outcomes for patients with NSCLC. Here we present the largest, to our knowledge, single-institution cohort of patients with NSCLC harboring MET exon 14 splicing mutations. These mutations occurred in $3.0 \%$ of nonsquamous NSCLCs, similar in prevalence to ALK translocations in our cohort and more common than rearrangements in ROS1, RET, and NTRK1 both in our cohort and in comparison with previously reported frequencies for these genomic alterations. ${ }^{30-35}$

Two recent reports have focused attention on MET exon 14 mutations as a targetable alteration in lung cancer. Paik et $\mathrm{al}^{13}$ described eight patients with MET exon 14 mutations, four of whom were treated with either crizotinib or cabozantinib, and partial responses were observed in some of these patients. Comparisons of clinical characteristics of these eight patients with those of patients with other molecular NSCLC subtypes were not provided given the sample size. Frampton et $\mathrm{al}^{12}$ impressively screened more than 38,000 tumors and identified 221 patient cases with MET exon 14 mutations; however, limited clinical and histopathologic data were available in this study. Here we describe detailed clinical, pathologic, and genomic features of 28 lung cancers harboring MET exon 14 mutations.

Uniquely, the MET exon 14 mutation seems to occur in older adults, with a median age of 72.5 years. This is the first time that a lung cancer mutation has been identified specifically in an older population and is in contrast to $A L K$ and ROS1 rearrangements, which tend to occur at younger ages of 50 to 60 years, ${ }^{30,36-38}$ and to KRAS, EGFR, and $B R A F$ mutations, which tend to occur at the ages of 61 to 66 years. ${ }^{38,39}$ Older patients may not be able to receive full-dose chemotherapy because of comorbidities; therefore, successful identification of targetable mutations in this population may improve treatment tolerability.

EGFR-, ALK-, and ROS1-driven cancers tend to occur in light or never-smokers. ${ }^{30,36-38}$ However, in our cohort, $64 \%$ of patients with MET exon 14 mutations had a history of tobacco use. We also found that although MET exon 14 mutations occurred predominantly in adenocarcinomas, $14 \%$ of patients with MET exon 14 mutations had pleomorphic (including sarcomatoid) histology, which is a higher-than-expected rate compared with historical case series. ${ }^{40}$ These findings highlight the need for comprehensive 

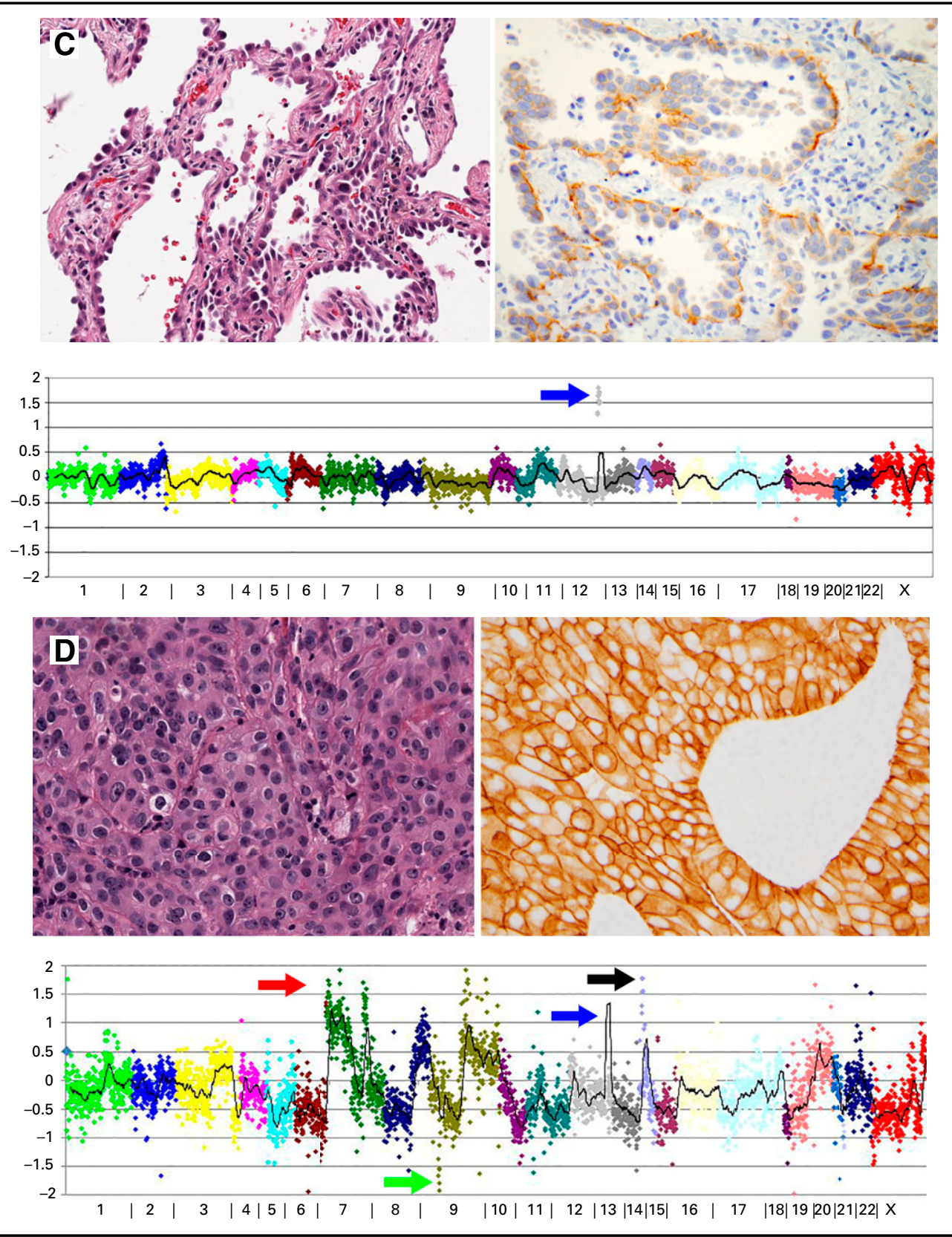

Fig 4. (continued) Representative (C) stage I and (D) stage IV cancers are shown. (C) Stage I minimally invasive lung adenocarcinoma (top left) associated with lowlevel c-Met protein expression (top right), focal amplification of MDM2 (bottom; arrow), and no other significant amplifications or deletions. (D) A small insertiondeletion event at the $3^{\prime}$ end of MET exon 14 was found in the context of a high-grade solid adenocarcinoma (top left) with highlevel c-Met protein expression (top right). Notably, this tumor showed evidence for significant genomic instability (bottom) marked by numerous copy-number alterations, including low-level amplification of EGFR and MET (estimated at approximately six copies; red arrow), low-level gain of $8 q$ (dark blue shading of points on chromosome 8q), homozygous deletion of $C D K N 2 A / B$ (green arrow), MDM2 amplification (blue arrow), and NKX2-1 amplification (black arrow). molecular profiling in all patients with NSCLC regardless of histology or clinical characteristics.

Nearly half of the patients with MET exon 14-mutated cancer in our study presented with stage I disease; this is in contrast to $A L K$ rearranged lung cancer, for example, which is rare in early-stage NSCLC. ${ }^{36}$ In The Cancer Genome Atlas study of lung adenocarcinoma $^{41}$ and in a separate Japanese study, ${ }^{42}$ MET amplification and $M E T$ exon 14 mutations seemed to be mutually exclusive in NSCLC; however, both of these studies used surgically resected (earlystage) lung tumors for analysis. Compared with patients presenting with METexon 14-mutated stage IA to IIIB NSCLC, patients with MET exon 14 mutations with stage IV disease in our study were significantly more likely to have concurrent MET genomic amplification and strong
c-Met expression. Our findings suggest that a MET exon 14 mutation may be an early event in lung tumorigenesis, and the stepwise addition of MET amplification and/or overexpression may contribute to a more aggressive clinical phenotype, but comparisons of serial tumor samples from the same patient will be necessary to validate this hypothesis.

Several different mutation types in MET exon 14 and its flanking introns were detected in our study, and this large degree of variation will have to be taken into account when designing clinical diagnostic sequencing assays to capture all possible activating MET mutations. Although some mutations affected splice acceptor and splice donor sites, which would easily be predicted to affect splicing, others occurred deeper within intron 13 and did not overlap with the splice acceptor site. In addition, a recurrent point mutation at the last 
A

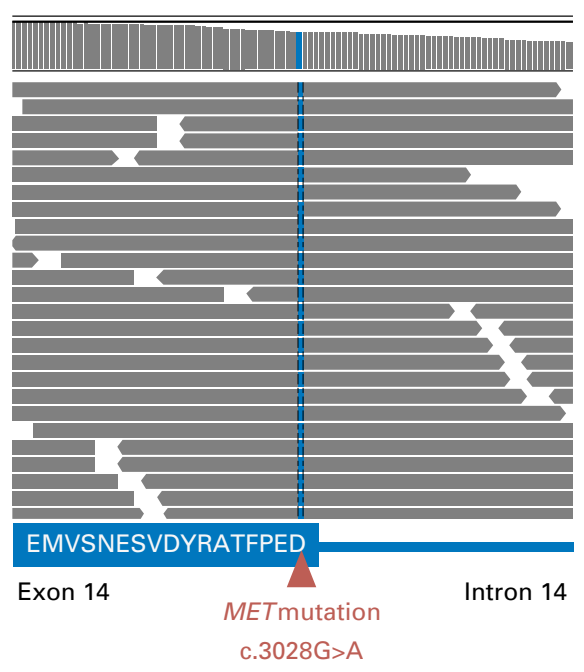

B

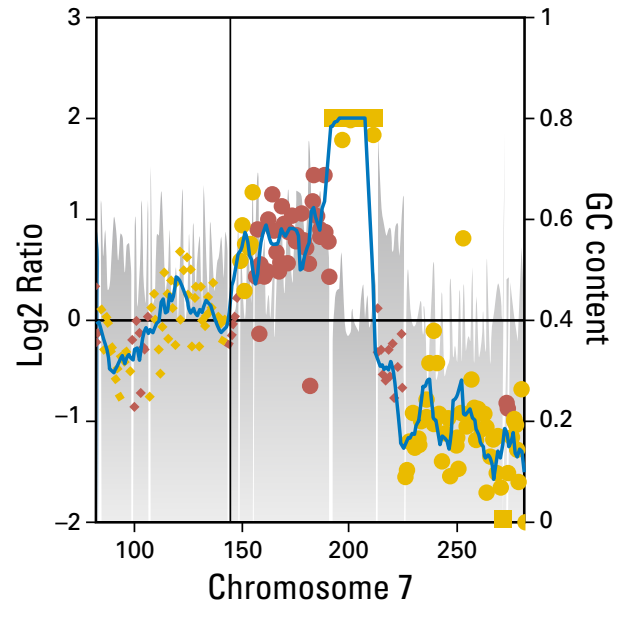

MET amplification approximately 18 copies MET:chr7 ratio $=8$
C

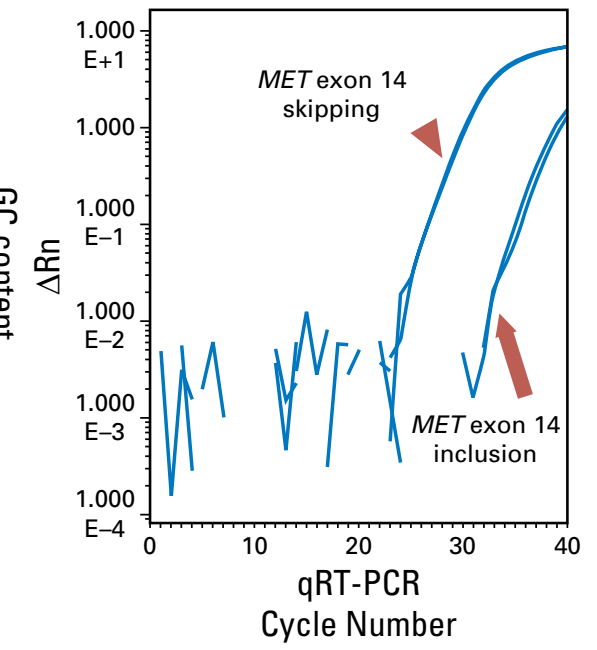

D

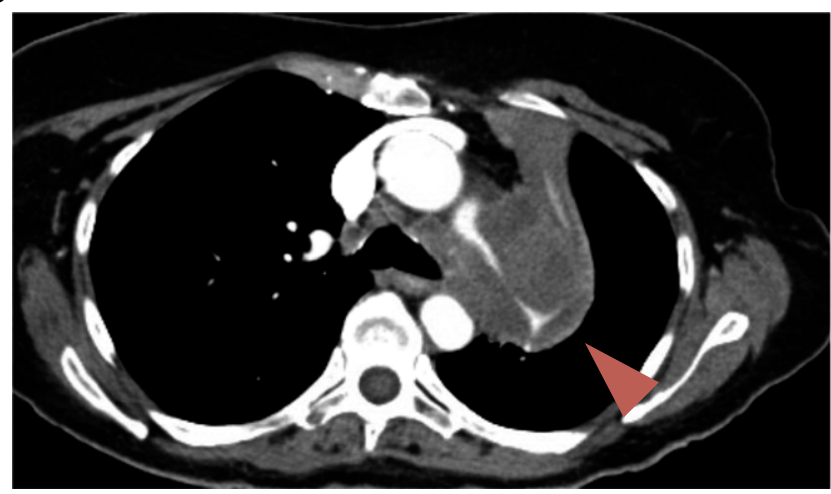

Pretreatment
E

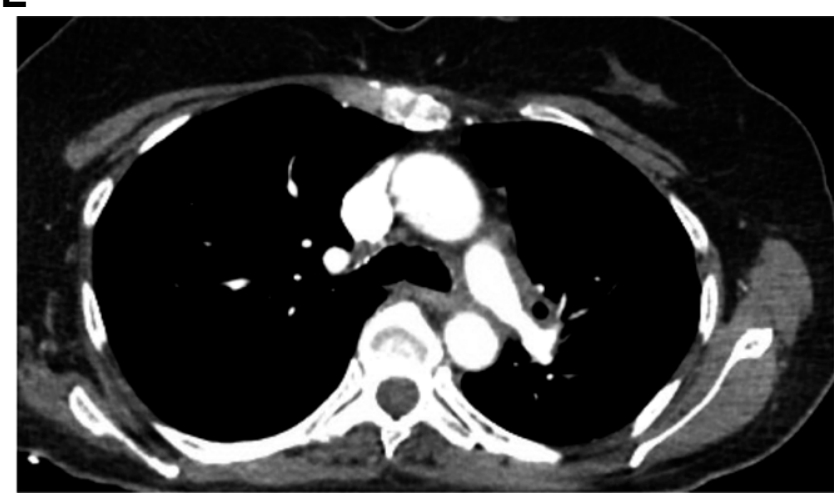

During crizotinib treatment (at 2 months)

Fig 5. Response to crizotinib in a patient with MET exon 14 mutation and concurrent MET amplification. A 64-year-old female never-smoker (patient 15) with poorly differentiated stage IV lung adenocarcinoma was found by next-generation sequencing to have (A) a MET exon $14 \mathrm{c} .3028 \mathrm{G}>\mathrm{A}$ mutation, (B) high-level MET amplification with a ratio of MET to chromosome 7 (chr7) of 8, and (C) exon 14 skipping (arrowhead), as detected by a real-time polymerase chain reaction (RT-PCR) -based assay. (C) Amplification of the wild-type MET transcript was also detected but at a higher RT-PCR cycle number (arrow). A chest computed tomography scan (axial view) at the level of the carina and left main pulmonary artery is shown (D) before and (E) after treatment with the c-Met inhibitor crizotinib.

nucleotide of exon 14 (c.3028G $>$ A) was detected in three patients; $\mathrm{G}>\mathrm{A}$ mutations in the last nucleotide of exons have been reported to cause alternative splicing in a number of other diseases. ${ }^{43-45}$ Robust exon 14 skipping was observed in 23 of 24 samples from patients with MET exon 14-mutant NSCLC tested using a qRT-PCR-based assay, demonstrating that a variety of sequence changes in this region can affect precursor mRNA processing. The only patient in whom exon 14 skipping was not demonstrated was the one patient with a c. $3008 \mathrm{~A}>\mathrm{G}$ point mutation, resulting in a Y1003C amino acid substitution. Tyrosine 1003 is necessary for the binding of $\mathrm{c}-\mathrm{Cbl}$ to c-Met and is required for receptor ubiquitination and degradation. ${ }^{16}$ In vitro, a Y1003F substitution mutation has been shown to transform fibroblasts in the absence of ligand, promote epithelialto-mesenchymal transition, and lead to cell dispersal. ${ }^{16}$ In this particular patient, the $\mathrm{Y} 1003 \mathrm{C}$ mutation, rather than exon 14 skipping, may have been the main mechanism of c-Met activation.

We describe a patient with a c.3028G $>$ A mutation causing MET exon 14 skipping who also had concurrent high-level amplification of the mutated MET allele and experienced a major partial response to the c-Met inhibitor crizotinib; no rearrangements in $A L K$ or ROS1, other targets of crizotinib, were detected in this patient. Whether sensitivity to crizotinib in this patient was conferred more by the MET exon 14 mutation or by MET amplification is unclear; however, responses to c-Met inhibitors have been reported in patients with MET exon 14 mutations without MET amplification. ${ }^{12,13}$ Prospective clinical trials will be necessary to determine if certain MET exon 14 mutations are more responsive to c-Met inhibition than others and whether concurrent $M E T$ amplification predicts for increased 
sensitivity to c-Met inhibitors. Studying how these initially responsive cancers acquire resistance to c-Met inhibitors will also be critical for the development of therapeutic strategies to overcome resistance. $^{46}$

\section{AUTHORS' DISCLOSURES OF POTENTIAL CONFLICTS} OF INTEREST

Disclosures provided by the authors are available with this article at www.jco.org.

\section{AUTHOR CONTRIBUTIONS}

Conception and design: Mark M. Awad, Geoffrey R. Oxnard, Suman Verma, Lynette M. Sholl

Collection and assembly of data: Mark M. Awad, David M. Jackman, Daniel O. Savukoski, Dimity Hall, Priyanka Shivdasani, Jennifer C. Heng, Lynette M. Sholl

Data analysis and interpretation: Mark M. Awad, Geoffrey R. Oxnard, Daniel O. Savukoski, Dimity Hall, Suzanne E. Dahlberg, Pasi A. Jänne, James Christensen, Peter S. Hammerman, Lynette M. Sholl

Manuscript writing: All authors Final approval of manuscript: All authors

\section{REFERENCES}

1. Lynch TJ, Bell DW, Sordella R, et al: Activating mutations in the epidermal growth factor receptor underlying responsiveness of non-small-cell lung cancer to gefitinib. N Engl J Med 350:2129-2139, 2004

2. Paez JG, Jänne $P A$, Lee JC, et al: EGFR mutations in lung cancer: Correlation with clinical response to gefitinib therapy. Science 304: 1497-1500, 2004

3. Pao W, Miller $\mathrm{V}$, Zakowski $M$, et al: EGF receptor gene mutations are common in lung cancers from "never smokers" and are associated with sensitivity of tumors to gefitinib and erlotinib. Proc Natl Acad Sci USA 101:13306-13311, 2004

4. Kwak EL, Bang YJ, Camidge DR, et al: Anaplastic lymphoma kinase inhibition in non-small-cell lung cancer. N Engl J Med 363:1693-1703, 2010

5. Shaw AT, Kim DW, Mehra R, et al: Ceritinib in ALK-rearranged non-small-cell lung cancer. N Engl J Med 370:1189-1197, 2014

6. Seto $T$, Kiura K, Nishio $M$, et al: $\mathrm{CH} 5424802$ (RO5424802) for patients with ALK-rearranged advanced non-small-cell lung cancer (AF-001JP study): A single-arm, open-label, phase 1-2 study. Lancet Oncol 14:590-598, 2013

7. Shaw AT, Ou SH, Bang YJ, et al: Crizotinib in ROS1-rearranged non-small-cell lung cancer. N Engl $J$ Med 371:1963-1971, 2014

8. Davies KD, Le AT, Theodoro MF, et al: Identifying and targeting ROS1 gene fusions in non-small cell lung cancer. Clin Cancer Res 18:4570-4579, 2012

9. Jenkins RW, Oxnard GR, Elkin S, et al: Response to crizotinib in a patient with lung adenocarcinoma harboring a MET splice site mutation. Clin Lung Cancer 16:e101-e104, 2015

10. Wagar SN, Morgensztern D, Sehn J: MET mutation associated with responsiveness to crizotinib. J Thorac Oncol 10:e29-e31, 2015

11. Mendenhall MA, Goldman JW: MET-mutated NSCLC with major response to crizotinib. J Thorac Oncol 10:e33-e34, 2015

12. Frampton GM, Ali SM, Rosenzweig $M$, et al: Activation of MET via diverse exon 14 splicing alterations occurs in multiple tumor types and confers clinical sensitivity to MET inhibitors. Cancer Discov 5:850-859, 2015

13. Paik PK, Drilon A, Fan PD, et al: Response to MET inhibitors in patients with stage IV lung adenocarcinomas harboring MET mutations causing exon 14 skipping. Cancer Discov 5:842-849, 2015

14. Camidge DR, Ou S-HI, Shapiro G, et al: Efficacy and safety of crizotinib in patients with advanced
c-MET-amplified non-small cell lung cancer (NSCLC). J Clin Oncol 32:506s, 2014 (suppl; abstr 8001)

15. Sakai K, Aoki S, Matsumoto K: Hepatocyte growth factor and Met in drug discovery. J Biochem 157:271-284, 2015

16. Peschard $P$, Fournier TM, Lamorte $L$, et al: Mutation of the $\mathrm{c}-\mathrm{Cb}$ TKB domain binding site on the Met receptor tyrosine kinase converts it into a transforming protein. Mol Cell 8:995-1004, 2001

17. Ma PC, Jagadeeswaran $R$, Jagadeesh $S$, et al: Functional expression and mutations of $\mathrm{c}-\mathrm{Met}$ and its therapeutic inhibition with SU11274 and small interfering RNA in non-small cell lung cancer. Cancer Res 65:1479-1488, 2005

18. Kong-Beltran M, Seshagiri $S$, Zha J, et al: Somatic mutations lead to an oncogenic deletion of met in lung cancer. Cancer Res 66:283-289, 2006

19. MacConaill LE, Garcia $E$, Shivdasani $P$, et al: Prospective enterprise-level molecular genotyping of a cohort of cancer patients. J Mol Diagn 16:660-672, 2014

20. Wagle N, Berger MF, Davis MJ, et al: Highthroughput detection of actionable genomic alterations in clinical tumor samples by targeted, massively parallel sequencing. Cancer Discov 2:82-93, 2012

21. Travis WD, Brambilla $E$, Noguchi $M$, et al: International Association for the Study of Lung Cancer/American Thoracic Society/European Respiratory Society international multidisciplinary classification of lung adenocarcinoma. J Thorac Oncol 6: 244-285, 2011

22. Travis WD, Brambilla E, Müller-Hermelink HK, et al: WHO Classification of Tumours of the Lung, Pleura, Thymus and Heart (ed 4). Lyon, France, International Agency for Research on Cancer, 2004

23. Gruver AM, Liu L, Vaillancourt $P$, et al: Immunohistochemical application of a highly sensitive and specific murine monoclonal antibody recognising the extracellular domain of the human hepatocyte growth factor receptor (MET). Histopathology 65:879-896, 2014

24. Sholl LM, Weremowicz S, Gray SW, et al: Combined use of ALK immunohistochemistry and FISH for optimal detection of ALK-rearranged lung adenocarcinomas. J Thorac Oncol 8:322-328, 2013

25. Cutz JC, Craddock KJ, Torlakovic E, et al: Canadian anaplastic lymphoma kinase study: A model for multicenter standardization and optimization of ALK testing in lung cancer. J Thorac Oncol 9:1255-1263, 2014

26. Sholl LM, Sun $H$, Butaney $M$, et al: ROS1 immunohistochemistry for detection of ROS1rearranged lung adenocarcinomas. Am J Surg Pathol 37:1441-1449, 2013
27. Liu $X$, Jia $Y$, Stoopler MB, et al: Nextgeneration sequencing of pulmonary sarcomatoid carcinoma reveals high frequency of actionable $M E T$ gene mutations. J Clin Oncol doi: JCO.2015.62.0674

28. Human Protein Reference Database. Hepatocyte growth factor receptor. http://www.hprd.org/ summary? hprd_id=01280\&isoform_id=01280_ 2\&isoform_name=Isoform_1

29. Oliner JD, Kinzler KW, Meltzer PS, et al: Amplification of a gene encoding a p53-associated protein in human sarcomas. Nature 358:80-83, 1992

30. Bergethon K, Shaw AT, Ou SH, et al: ROS1 rearrangements define a unique molecular class of lung cancers. J Clin Oncol 30:863-870, 2012

31. Lipson D, Capelletti M, Yelensky R, et al: Identification of new ALK and RET gene fusions from colorectal and lung cancer biopsies. Nat Med 18: 382-384, 2012

32. Kohno $T$, Ichikawa $H$, Totoki $Y$, et al: KIF5BRET fusions in lung adenocarcinoma. Nat Med 18: 375-377, 2012

33. Takeuchi $K$, Soda $M$, Togashi $Y$, et al: RET, ROS1 and ALK fusions in lung cancer. Nat Med 18: 378-381, 2012

34. Wang $R, H u H$, Pan $Y$, et al: RET fusions define a unique molecular and clinicopathologic subtype of non-small-cell lung cancer. J Clin Oncol 30: 4352-4359, 2012

35. Vaishnavi A, Capelletti $M$, Le AT, et al: Oncogenic and drug-sensitive NTRK1 rearrangements in lung cancer. Nat Med 19:1469-1472, 2013

36. Rodig SJ, Mino-Kenudson M, Dacic S, et al: Unique clinicopathologic features characterize ALKrearranged lung adenocarcinoma in the western population. Clin Cancer Res 15:5216-5223, 2009

37. Shaw AT, Yeap BY, Mino-Kenudson $M$, et al: Clinical features and outcome of patients with non-small-cell lung cancer who harbor EML4-ALK. $\mathrm{J}$ Clin Oncol 27:4247-4253, 2009

38. Paik PK, Arcila ME, Fara $M$, et al: Clinical characteristics of patients with lung adenocarcinomas harboring BRAF mutations. J Clin Oncol 29: 2046-2051, 2011

39. Cardarella S, Ogino A, Nishino M, et al: Clinical, pathologic, and biologic features associated with BRAF mutations in non-small cell lung cancer. Clin Cancer Res 19:4532-4540, 2013

40. Yendamuri S, Caty L, Pine M, et al: Outcomes of sarcomatoid carcinoma of the lung: a Surveillance, Epidemiology, and End Results Database analysis. Surgery 152:397-402, 2012

41. Cancer Genome Atlas Research Network: Comprehensive molecular profiling of lung adenocarcinoma. Nature 511:543-550, 2014 [Erratum: Nature 514:262, 2014] 
42. Onozato $R$, Kosaka $T$, Kuwano $H$, et al: Activation of MET by gene amplification or by splice mutations deleting the juxtamembrane domain in primary resected lung cancers. J Thorac Oncol 4: 5-11, 2009

43. Satokata I, Tanaka K, Yuba S, et al: Identification of splicing mutations of the last nucleotides of exons, a nonsense mutation, and a missense mutation of the XPAC gene as causes of group A xeroderma pigmentosum. Mutat Res 273:203-212, 1992

44. Kanai $N$, Yanai $F$, Hirose $S$, et al: $A$ G to $A$ transition at the last nucleotide of exon 6 of the gamma c gene (868G->A) may result in either a splice or missense mutation in patients with X-linked severe combined immunodeficiency. Hum Genet 104:36-42, 1999
45. Ozkara HA, Sandhoff K: A new point mutation ( $\mathrm{G} 412$ to $A$ ) at the last nucleotide of exon 3 of hexosaminidase alpha-subunit gene affects splicing Brain Dev 25:203-206, 2003

46. Lovly CM, Shaw AT: Molecular pathways: Resistance to kinase inhibitors and implications for therapeutic strategies. Clin Cancer Res 20: 2249-2256, 2014

\section{Let Cancer.Net Help You Address Clinical Trials With Your Patients}

Multiple barriers exist when it comes to patient participation in clinical trials. In response to these barriers, Cancer.Net now offers PRE-ACT (Preparatory Education About Clinical Trials). This FREE interactive video-based program provides patients with tailored clinical trial information in an effort to help them be better prepared to make an informed decision about clinical trial participation. Learn more at cancer.net/PREACT. 


\section{AUTHORS' DISCLOSURES OF POTENTIAL CONFLICTS OF INTEREST}

MET Exon 14 Mutations in Non-Small-Cell Lung Cancer Are Associated With Advanced Age and Stage-Dependent MET Genomic Amplification and c-Met Overexpression

The following represents disclosure information provided by authors of this manuscript. All relationships are considered compensated. Relationships are self-held unless noted. I = Immediate Family Member, Inst = My Institution. Relationships may not relate to the subject matter of this manuscript. For more information about ASCO's conflict of interest policy, please refer to www.asco.org/rwc or jco.ascopubs.org/site/ifc.

Mark M. Awad

Consulting or Advisory Role: Genetech, Merck, Pfizer, Boehringer Ingelheim, AbbVie, AstraZeneca

Geoffrey R. Oxnard

Honoraria: Chugai Pharmaceutical, Boehringer Ingelheim, AstraZeneca Consulting or Advisory Role: Boehringer Ingelheim, Clovis Oncology, AstraZeneca, Sysmex, ARIAD Pharmaceuticals

David M. Jackman

Consulting or Advisory Role: Genentech, Celgene

Daniel O. Savukoski

No relationship to disclose

Dimity Hall

No relationship to disclose

Priyanka Shivdasani

No relationship to disclose

Jennifer C. Heng

No relationship to disclose

Suzanne E. Dahlberg

Patents, Royalties, Other Intellectual Property: Patent pending for statistical model assessing tumor growth (Inst)

\section{Pasi A. Jänne}

Stock or Other Ownership: Gatekeeper Pharmaceuticals Consulting or Advisory Role: AstraZeneca, Roche, Pfizer Research Funding: AstraZeneca, Astellas Pharma

Patents, Royalties, Other Intellectual Property: Postmarketing royalties from Dana-Farber Cancer Institute-owned intellectual property of EGFR mutations licensed to LabCorp

\section{Suman Verma \\ Employment: ResearchDX \\ Company: ResearchDX}

\section{James Christensen}

Employment: Mirati Therapeutics

Leadership: Mirati Therapeutics

Stock or Other Ownership: Mirati Therapeutics

Peter S. Hammerman

Employment: Pfizer (I)

Stock or Other Ownership: Pfizer (I)

Consulting or Advisory Role: Janssen Oncology, AstraZeneca, Clovis Oncology, ARIAD Pharmaceuticals, Array BioPharma, MolecularMD Patents, Royalties, Other Intellectual Property: DDR2 mutations in squamous cell lung cancer; tumor suppressor and oncogene biomarkers predictive of anti-immune checkpoint inhibitor response

Lynette M. Sholl

Consulting or Advisory Role: Genentech 


\section{Appendix}

\section{Methods}

\section{Next-Generation Sequencing}

Manual macrodissection from unstained tissue sections was performed to enrich for areas containing $\geq 20 \%$ tumor nuclei. DNA was isolated following standard extraction protocols (Qiagen, Valencia, CA) and quantified using PicoGreen-based dsDNA detection (Life Technologies, Carlsbad, CA). Sequencing libraries were prepared from 50 ng of DNA using Illumina TruSeq LT reagents (Illumina, San Diego, CA) and enriched for exons and select introns in 282 genes implicated in cancer biology (Appendix Table A1) through solution-based hybrid capture using an Agilent SureSelect custom RNA bait set (Agilent Technologies, Santa Clara, CA). Massively parallel sequencing performed on Illumina HiSeq2500 with $100 \times 100$ paired-end reads achieved an average mean target coverage of $187 \times$ per sample.

Pooled sample reads were demultiplexed and sorted, and duplicate reads were removed using Picard. Reads were aligned to the reference sequence b37 edition from the Human Genome Reference Consortium using BWA software (Li H, et al: Bioinformatics 25:1754-1760, 2009). Mutation analysis for single-nucleotide variants was performed using MuTect (version 10.27200 ; Cibulskis K, et al: Nat Biotechnol 31:213-219, 2013) and annotated using Oncotator software (http://www.broadinstitute.org/oncotator). Insertions and deletions were called using Indelocator software (https:/www.broadinstitute.org/cancer/cga/indelocator). Gene rearrangements were identified using BreaKmer (Abo RP, et al: Nucleic Acids Res 43:e19, 2015). Integrative Genomics Viewer (version 2.0.16 or later; https://www.broadinstitute.org/igv) was used for visualization and interpretation. Variants present at a population frequency of greater than $0.1 \%$ in the Exome Sequencing Project database were filtered out as germline polymorphisms.

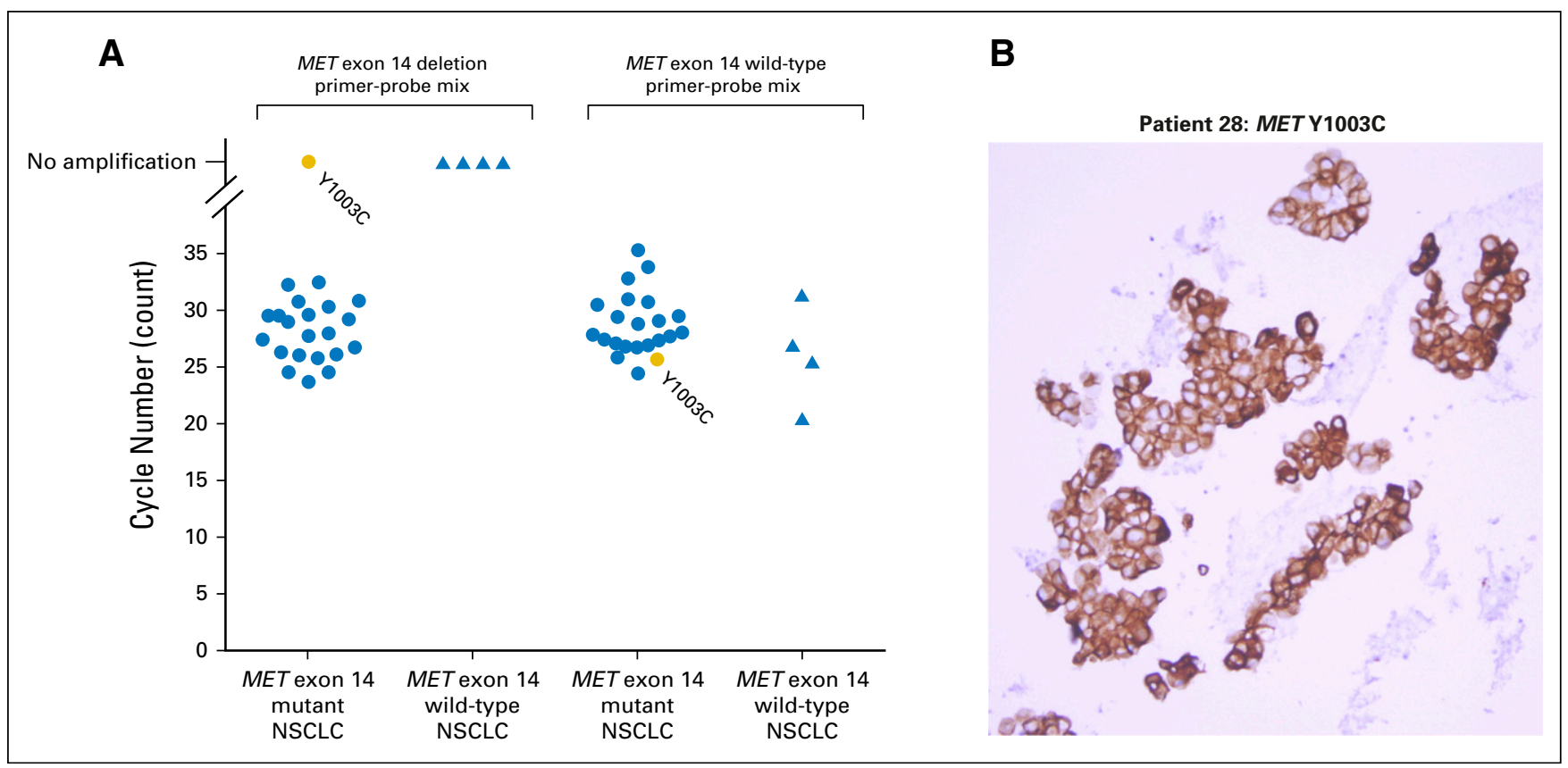

Fig A1. Of the 28 patients in this cohort with MET exon 14 mutations, 24 had RNA available for testing. MET exon 14 skipping was detected in 23 (96\%) of 24 patients

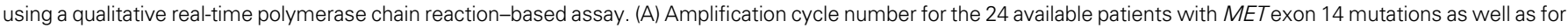

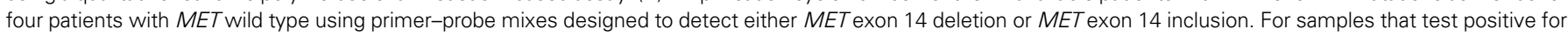

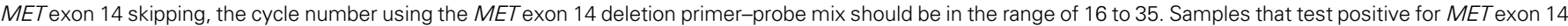

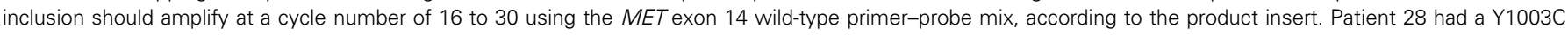

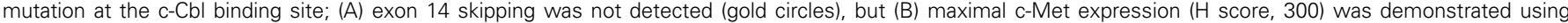
immunohistochemistry. NSCLC, non-small-cell lung cancer. 
Features of NSCLC With MET Exon 14 Mutations

\begin{tabular}{|c|c|c|c|c|c|c|c|c|c|}
\hline \multicolumn{10}{|c|}{ Gene } \\
\hline$A B L 1$ & BRCA1 & CEBPA & ESR1 & GATA3 & LMO1 & $N B N$ & PRAME & SDHAF2 & TCF3 \\
\hline$A K T 1$ & $B R C A 2$ & CHEK2 & ETV1 & GATA4 & LMO2 & NF1 & PRDM1 & $S D H B$ & TCF7L1 \\
\hline AKT2 & $B R D 4$ & CIITA & ETV4 & GATAG & LMO3 & NF2 & PRF1 & $S D H C$ & TCF7L2 \\
\hline AKT3 & $B R I P 1$ & CREBBP & ETV5 & GL/1 & MAP2K1 & NFE2L2 & PRKAR1A & $S D H D$ & TERT \\
\hline$A L K$ & $B \cup B 1 B$ & $C R K L$ & ETV6 & GLI2 & MAP2K 4 & NFKBIA & PRKCl & SETBP1 & TET2 \\
\hline$A L O X 12 B$ & CARD11 & CRLF2 & EWSR1 & GL/3 & MAP3K 1 & NFKBIZ & PRKCZ & SETD2 & TNFAIP3 \\
\hline$A P C$ & $C B L$ & CRTC1 & EXT1 & GNA11 & MAPK1 & $N K \times 2-1$ & PRKDC & SF1 & TRA \\
\hline$A R$ & $C B L B$ & CRTC2 & EXT2 & $G N A Q$ & MCL1 & NOTCH1 & PRPF $40 B$ & SF3B1 & TRB \\
\hline$A R A F$ & CCND1 & CTNNB1 & $E Z H 2$ & GNAS & MDM2 & $\mathrm{NOTCH} 2$ & PRPF8 & SH2B3 & TRG \\
\hline$A R I D 1 A$ & CCND2 & CUX1 & FAM46C & GPC3 & MDM4 & NPM1 & PSMD13 & SMAD2 & TSC1 \\
\hline$A R I D 1 B$ & CCND3 & $C Y L D$ & FANCA & GSTM5 & MECOM & NRAS & PTCH1 & TP53 & TSC2 \\
\hline$A R I D 2$ & CCNE1 & $D D B 2$ & FANCC & H3F3A & MEF2B & NTRK1 & PTEN & TRA & U2AF1 \\
\hline$A S X L 1$ & $C D 274$ & DDR2 & FANCD2 & HNF1A & MEN1 & NTRK2 & PTK2 & SMARCB1 & VHL \\
\hline ATM & CD58 & DICER1 & FANCE & HRAS & MET & NTRK3 & PTPN11 & SMC1A & WRN \\
\hline ATRX & $C D 79 B$ & DIS3 & FANCF & ID3 & MITF & PALB2 & SMAD4 & SMC3 & WT1 \\
\hline AURKA & $C D C 73$ & $D M D$ & FANCG & $I D H 1$ & $M L H 1$ & PARK2 & SMARCA4 & SMO & $X P A$ \\
\hline$A \cup R K B$ & $\mathrm{CDH} 1$ & DNMT3A & FAS & $\mathrm{IDH} 2$ & $M L L$ & PAX5 & $R A D 21$ & SOCS1 & $X P C$ \\
\hline$A X L$ & $C D K 1$ & $E G F R$ & FBXW7 & IGF1R & $M L L 2$ & PDCD1LG2 & $R A F 1$ & SOX2 & XPO1 \\
\hline$B 2 M$ & $C D K 2$ & EP300 & FGFR1 & $\lg H$ & $M P L$ & PDGFRA & $R A R A$ & SOX9 & ZNF217 \\
\hline$B A P 1$ & $C D K 4$ & EPHAЗ & FGFR2 & $\lg L$ & MSH2 & PDGFRB & $R B 1$ & $S R C$ & ZNF708 \\
\hline$B C L 2$ & $C D K 5$ & EPHA5 & FGFR3 & IKZF1 & MSH6 & PHFG & $R B L 2$ & SRSF2 & ZRSR2 \\
\hline$B C L 2 L 1$ & $C D K 6$ & EPHA7 & FGFR4 & IKZF3 & MTOR & PHOX2B & $R E L$ & STAG1 & \\
\hline$B C L 2 L 12$ & $C D K 9$ & ERBB2 & $\mathrm{FH}$ & $J A K 2$ & MUTYH & PIK3C2B & $R E T$ & STAG2 & \\
\hline$B C L 6$ & CDKN1A & $E R B B 3$ & FKBPG & $J A K 3$ & $M Y B$ & PIKЗCA & RFWD2 & STAT3 & \\
\hline$B C O R$ & $C D K N 1 B$ & ERBB4 & FLCN & KDM6A & $M Y B L 1$ & PIK3R1 & RHPN2 & STAT6 & \\
\hline BCORL1 & CDKN1C & ERCC2 & FLT1 & $K D M 6 B$ & $M Y C$ & PIM1 & ROS1 & STK11 & \\
\hline$B L M$ & CDKN2A & ERCC3 & FLT3 & $K D R$ & MYCL1 & PMS1 & RPL26 & SUFU & \\
\hline$B M P R 1 A$ & $C D K N 2 B$ & ERCC4 & FLT4 & $K I T$ & MYCN & PMS2 & $R \cup N \times 1$ & SUZ12 & \\
\hline$B R A F$ & CDKN2C & ERCC5 & FUS & KRAS & MYD88 & PNRC1 & $S B D S$ & $S Y K$ & \\
\hline
\end{tabular}

Table A2. Immunohistochemical Antibodies Used in This Study

\begin{tabular}{|c|c|c|c|c|}
\hline Antibody & Clone (Company) & Dilution & Antigen Retrieval & Detection System \\
\hline MET & SP44 (Spring Bioscience, Pleasanton, CA) ${ }^{23}$ & $1: 100$ & Citrate buffer/pressure cooker & Leica Novolink \\
\hline ALK & 5A4 (Leica Biosystems, Nussloch, Germany) 24,25 & $1: 75$ & Citrate buffer/pressure cooker & Leica Novolink \\
\hline ROS1 & D4D6 (Cell Signaling Technology, Danvers, MA) ${ }^{26}$ & $1: 400$ & EDTA/pressure cooker & Leica Novolink \\
\hline
\end{tabular}

